\title{
Aging of Oxygen-Treated Trimethylsilane Plasma-Polymerized Films Using Spectroscopic Ellipsometry
}

\author{
Taher M. El-Agez, ${ }^{1}$ David M. Wieliczka, ${ }^{2}$ Chris Moffitt, ${ }^{2}$ and Sofyan A. Taya ${ }^{1}$ \\ ${ }^{1}$ Physics Department, Islamic University of Gaza, P.O. Box 108, Gaza, Palestine \\ ${ }^{2}$ Physics Department, University of Missouri-Kansas City, Kansas City, MO 64110, USA
}

Correspondence should be addressed to Sofyan A. Taya, staya@iugaza.edu.ps

Received 6 June 2011; Accepted 2 August 2011

Academic Editor: Edward E. Eyler

Copyright ( 2011 Taher M. El-Agez et al. This is an open access article distributed under the Creative Commons Attribution License, which permits unrestricted use, distribution, and reproduction in any medium, provided the original work is properly cited.

Oxygen-treated trimethylsilane (TMS) plasma-polymerized films are investigated using rotating polarizer and analyzer ellipsometer. Aging process and composition of the samples are studied. Coordinated X-ray photoelectron spectroscopy (XPS) depth profiling studies on these films is presented for more detailed understanding of the aging process as well as the modeling of these films.

\section{Introduction}

Amorphous hydrogenated silicon-carbon thin films have received an increasing interest in the development of optoelectronic devices and in the metallurgical field as a hard protective coating. Low-temperature plasma is one of the many different methods to deposit such films on metals and semiconductors. The interaction between the monomer vapor and the electromagnetic energy can be achieved in different ways. The direct current (DC) approach has proven to be an efficient method in coatings on metals for corrosion protection purposes. The conductive substrate on which the film is being deposited plays the role as the cathode of the DC glow discharge. An enhanced magnetic anode (magnetron) is used to confine and focus the plasma onto the coated substrate. The mechanism of the polymerization can be explained based on the concept of competitive ablation and polymerization [1]. The plasma parameters determine the outcome of the polymerization process to be either etching (ablation) or plasma polymerization.

Ellipsometry is a powerful tool for optical characterization of thin films [2-9]. It is used for determining optical properties of materials, thicknesses, porosity, and crystal orientation. The principle of operation of ellipsometry is based on measuring polarization changes occurring upon reflection at oblique incidence of a polarized plane wave [2].
The basic quantities measured with an ellipsometer are the ellipsometric parameters $\psi$ and $\Delta$ from which the optical parameters of a structure can be extracted by inverting Fresnel equations [6].

The main interest in this study is to investigate the aging process of oxygen-treated TMS $\left(\left(\mathrm{CH}_{3}\right)_{3} \mathrm{SiH}\right)$ plasmapolymerized films using one to two rotating polarizer and analyzer ellipsometer. These kinds of films are very hard to analyze due to the complexity of this problem as well as the wide range of parameters and variables involved. Similar films are believed to be porous, amorphous, macroscopically homogenous, and microscopically heterogeneous. They are composed of silicon, carbon, oxygen, hydrogen, and occasionally nitrogen and fluorine. Therefore, this work is a preliminary trial to understand the aging and composition of these films.

In this work, single wavelength as well as spectroscopic ellipsometry will be used to study the aging of these films prepared by low-temperature DC plasma polymerization of TMS. Coordinated XPS depth profiling studies on these films will be presented for a more detailed understanding of the aging process as well as the modeling of these films. The change in the dielectric function of similar films is known [9-12]. Dramatic changes occur in fresh samples within the first few hours of exposure to atmospheric conditions. 

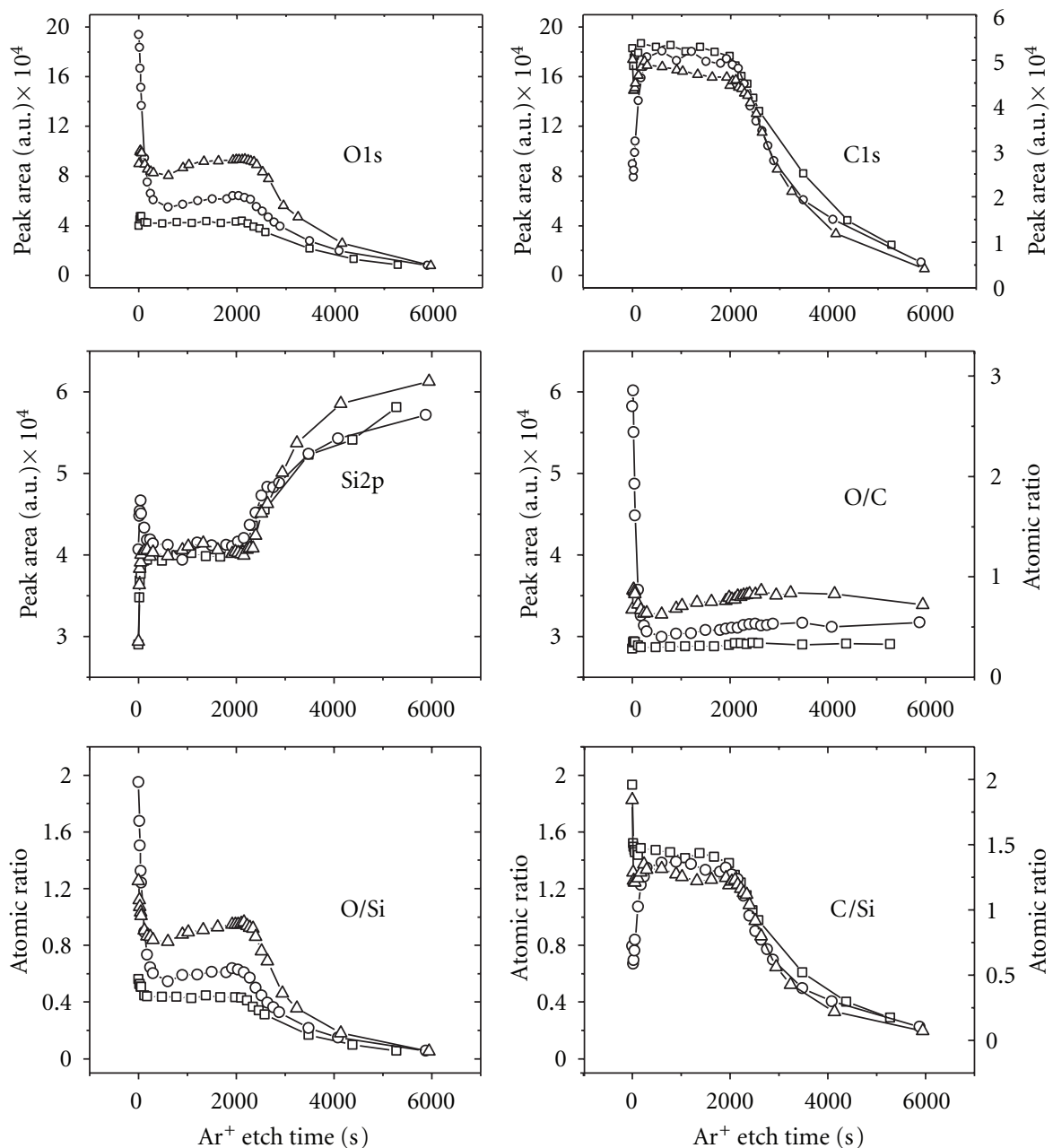

$$
\begin{aligned}
& -\square \text { TMS on Si after } 1 \text { day } \\
& --\mathrm{O}_{2} \text { treated after } 60 \text { days } \\
& - \text { TMS on } \mathrm{Si} \text {, after } 80 \text { days }
\end{aligned}
$$

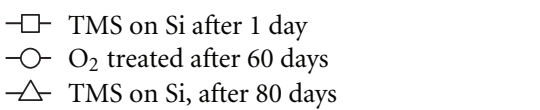

FIGURE 1: XPS sputter depth profile comparing aging of oxygen-treated TMS sample and natural aging of plain TMS plasma polymer.

\section{Experimental Work}

The samples under consideration have been prepared at the Center for Surface Science and Plasma Technology, University of Missouri Columbia, USA. The measurements have been carried out at Physics Department, University of Missouri-Kansas City, Kansas City, Mo, USA.

The films are prepared by mounting (using conductive silver paint) clean small (111) single crystal silicon pieces on an aluminum panel, so many samples were made with each deposition under the same conditions. The panel is then connected to the cathode of a low-temperature DC plasma chamber. TMS gas is used as a monomer during the plasma polymerization process. The following plasma parameters were used, 5 watts plasma power, 1 minute and 40 seconds deposition time, 50 millitorr pressure, and $1 \mathrm{sccm}$ flow rate of TMS gas. Then, TMS is turned off, while dry oxygen is turned on for 1 minute with 2 watts of plasma power, 50 millitorr pressure, and $1 \mathrm{sccm}$ oxygen flow rate. The treatment process helps to cure the top surface by oxidization of the unsaturated free radicals. XPS depth profile measurements on oxygen-treated TMS films revealed the presence of a very thin distinguishable layer on top of the usual TMS film as shown in Figures 1 and 2. Therefore, a three-layer model shown in Figure 3 is suggested to analyze the ellipsometric data taken on these films. The XPS depth profile measurements showed a significant increase in the atomic oxygen signal and a considerable decrease in the carbon atomic signal within the top layer as shown clearly in Figure 1. Surprisingly, the total silicon XPS atomic concentration has increased compared to the rest of the TMS bulk material. This might be explained based on the depletion of carbon atoms from the top layer during the oxygen treatment process and the formation of a siliconoxygen-rich region. The $\mathrm{O} / \mathrm{Si}$ ratio decays from $2 / 1$ at the air/film interface to $0.6 / 1$ at the top film/bulk TMS interface. On the other hand, the $\mathrm{C} / \mathrm{Si}$ ratio grows from $0.5 / 1$ at the air/film interface to $1.25 / 1$ ratio at the top film/bulk TMS interface. 

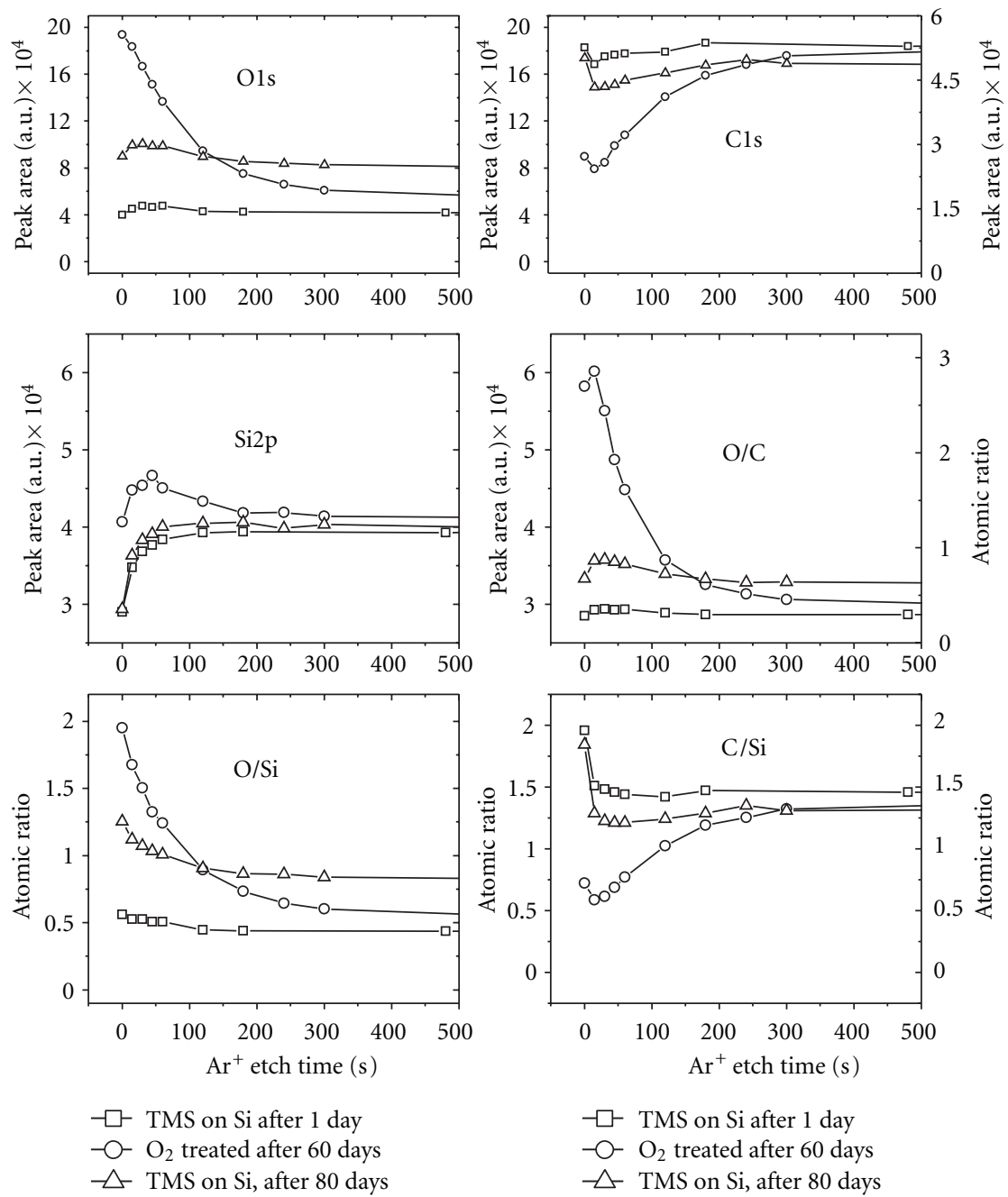

FIGURE 2: Enlarged XPS sputter depth profile comparing aging of oxygen-treated TMS sample and natural aging of plain TMS plasma polymer.

\begin{tabular}{|c|}
\hline Oxidized layer \\
\hline Trimethylsilane (TMS) film \\
\hline Silicon dioxide $(4 \mathrm{~nm})$ \\
\hline \\
Silicon substrate \\
\end{tabular}

FIGURE 3: Suggested model for oxygen-treated TMS film on silicon wafer.

A home-made rotating polarizer and analyzer ellipsometer (RPAE) in which the polarizer and the analyzer rotate with a speed ratio of $1: 2$ was used in this work [13].

Single wavelength ellipsometric measurements are carried out at $632.8 \mathrm{~nm}$ and incidence angle of $70^{\circ}$. The native oxide on the silicon substrate is measured to be $4 \mathrm{~nm}$ prior to the film deposition and is assumed to be intact during the polymerization process. The inversion of the ellipsometric
TABLE 1: The index and thickness of both the top layer and the TMS film of oxygen-treated plasma-polymerized film at $632.8 \mathrm{~nm}$.

\begin{tabular}{lcccc}
\hline Day & \multicolumn{2}{c}{ Top layer } & \multicolumn{2}{c}{ TMS film } \\
Thickness $(\mathrm{nm})$ & Index & Thickness $(\mathrm{nm})$ \\
\hline 1 & 1.51959 & 7.1 & 1.6815 & 95.4 \\
7 & 1.55123 & 8.1 & 1.659 & 95.7 \\
15 & 1.553 & 8.8 & 1.6465 & 96.3 \\
50 & 1.5799 & 13.7 & 1.624 & 93.9 \\
\hline
\end{tabular}

parameters $\psi$ and $\Delta$ taken at $632.8 \mathrm{~nm}$ showed that the top layer has doubled in thickness in a seven-week period, while the index has showed an increase of $4 \%$ in the same period. On the other hand, the thickness of the TMS layer has not encountered a significant change in the seven-week period, but the refractive index showed a $3.4 \%$ decrease in that period. The obtained results of the index and thickness for the top layer as well as the underlying TMS layer are listed in Table 1. 
TABLE 2: The effective medium approximation applied on oxygen-treated TMS films after aging for 1 day. The obtained atomic ratios are compared to XPS results.

\begin{tabular}{|c|c|c|c|c|c|c|c|c|c|}
\hline \multirow{2}{*}{$\lambda(\mathrm{nm})$} & \multirow{2}{*}{$n_{\text {eff }}$} & \multirow{2}{*}{$n(\mathrm{a}-\mathrm{SiC}: \mathrm{H})$} & \multirow{2}{*}{$n\left(\mathrm{a}-\mathrm{SiO}_{2}\right)$} & \multirow{2}{*}{$v_{1}$} & \multirow{2}{*}{$v_{2}$} & \multicolumn{2}{|c|}{$\mathrm{C} / \mathrm{Si}$} & \multicolumn{2}{|c|}{$\mathrm{O} / \mathrm{Si}$} \\
\hline & & & & & & XPS & EMA & XPS & EMA \\
\hline 700 & 1.672 & 1.753 & 1.455 & 0.55 & 0.45 & NA & 2 & NA & 0.56 \\
\hline 600 & 1.681 & 1.787 & 1.458 & 0.68 & 0.32 & NA & 1.85 & NA & 0.64 \\
\hline 500 & 1.709 & 1.82 & 1.462 & 0.7 & 0.3 & NA & 1.9 & NA & 0.62 \\
\hline 420 & 1.749 & 1.847 & 1.468 & 0.75 & 0.25 & NA & 2 & NA & 0.53 \\
\hline
\end{tabular}

TABLE 3: The effective medium approximation applied on oxygen-treated TMS films after aging for 50 days. The obtained atomic ratios are compared to XPS results after 50 days.

\begin{tabular}{|c|c|c|c|c|c|c|c|c|c|}
\hline \multirow{2}{*}{$\lambda(\mathrm{nm})$} & \multirow{2}{*}{$n_{\text {eff }}$} & \multirow{2}{*}{$n(\mathrm{a}-\mathrm{SiC}: \mathrm{H})$} & \multirow{2}{*}{$n\left(\mathrm{a}-\mathrm{SiO}_{2}\right)$} & \multirow{2}{*}{$v_{1}$} & \multirow{2}{*}{$v_{2}$} & \multicolumn{2}{|c|}{$\mathrm{C} / \mathrm{Si}$} & \multicolumn{2}{|c|}{$\mathrm{O} / \mathrm{Si}$} \\
\hline & & & & & & XPS & EMA & XPS & EMA \\
\hline 700 & 1.616 & 1.753 & 1.455 & 0.53 & 0.47 & 1.35 & 1.3 & 0.7 & 0.88 \\
\hline 600 & 1.623 & 1.787 & 1.458 & 0.52 & 0.48 & 1.35 & 1.2 & 0.7 & 0.93 \\
\hline 500 & 1.648 & 1.82 & 1.462 & 0.53 & 0.47 & 1.35 & 1.23 & 0.7 & 0.90 \\
\hline 420 & 1.676 & 1.847 & 1.468 & 0.55 & 0.45 & 1.35 & 1.47 & 0.7 & 0.80 \\
\hline
\end{tabular}

A suggested mechanism of the aging process of oxygentreated TMS films could be explained as follows: oxygen from all atmospheric sources (e.g., $\mathrm{H}_{2} \mathrm{O}, \mathrm{O}_{2}$, etc.) diffuses in through the porous fabric starting the oxidation of the underlying TMS material which can be seen to be the cause of the decrease in the refractive index of that layer. The oxidation process causes carbon compounds to diffuse out towards the top layer. The diffused carbon will add to the silicon oxygen network in the top layer, which can be seen to be the cause of the increase in the index of that layer. Therefore, the carbon content of the bulk TMS decreases with time, and in the mean time, it increases in the top layer. This process continues until equilibrium in the carbon and oxygen content between the top layer and the underlying TMS layer may occur.

The optical response ( $\left.n_{\text {eff }}\right)$ of the oxidized films can be roughly obtained by mixing the oxygen free a- $\mathrm{Si}_{0.25} \mathrm{C}_{0.75}: \mathrm{H}$ with amorphous silicon dioxide $\left(\mathrm{a}-\mathrm{SiO}_{2}\right)$ in certain proportions.

In order to try to understand the chemistry of aging of oxygen-treated TMS films, the following assumptions are made. First, the films are presumed to be macroscopically homogenous, while having a heterogeneous microscopic structure [14]. Second, the effective index of these films is modeled according to the Bruggemann effective medium approximation (EMA) [15]. The EMA is one of many simple effective medium theories that describe the optical properties of heterogeneous mixtures. For the EMA to be valid, few assumptions are made. First, the inclusions (the mixed entities) in the mixture are assumed to have independent polarizabilities, that is, they do not interact with each other. Second, the scale of heterogeneity is assumed to be much smaller than the wavelength of light in the medium, yet large enough so that the individual components have bulklike dielectric properties. Third, the mixed components are assumed to be embedded in a background with optical properties as the effective medium itself.

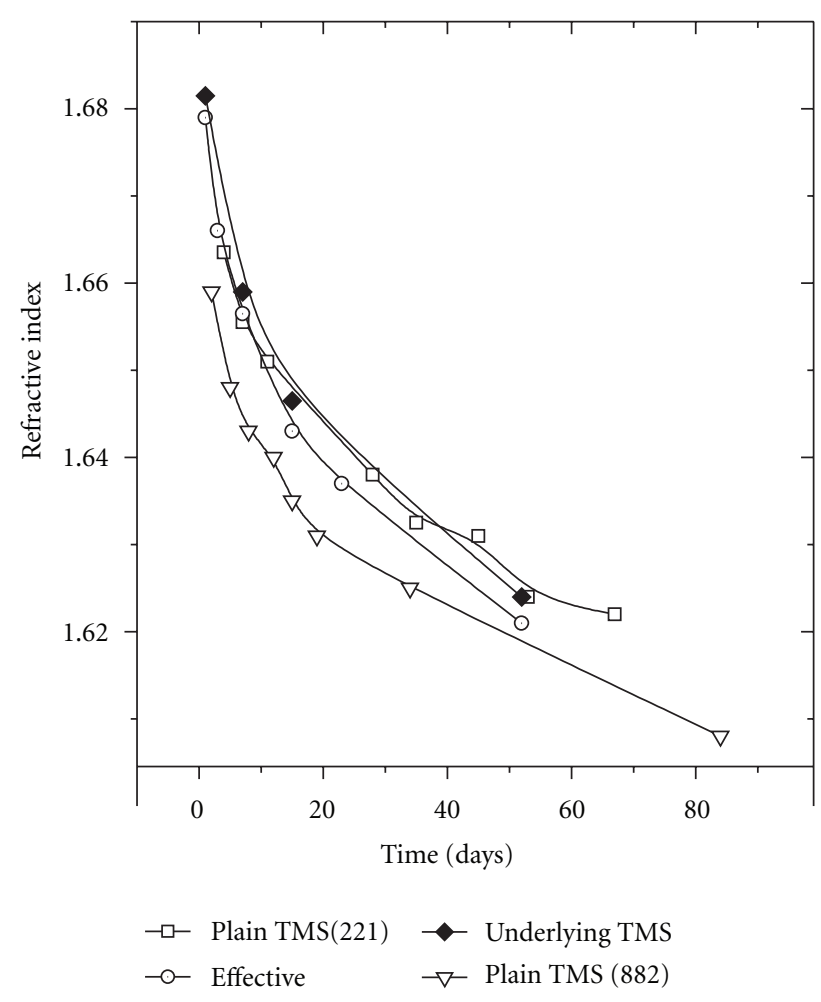

Figure 4: Comparison between plain TMS films and oxygentreated TMS films.

According to Bruggemann [15], the optical properties of the individual inclusions and the effective medium are related by

$$
\begin{gathered}
\sum_{j} v_{j} \frac{n_{j}^{2}-n_{\mathrm{eff}}^{2}}{n_{j}^{2}+2 n_{\mathrm{eff}}^{2}}=0, \\
\sum_{j} v_{j}=1,
\end{gathered}
$$




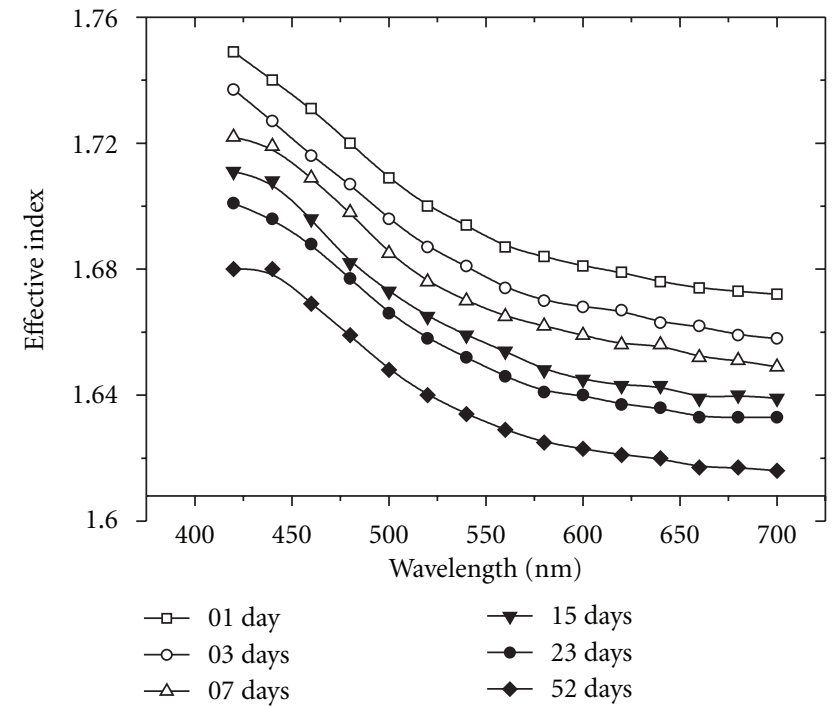

FIgURE 5: The behavior of the effective index of oxygen-treated plain TMS on silicon as a function of time and wavelength.

where $n_{j}$ and $n_{\text {eff }}$ are the refractive index of the inclusions and the effective medium, respectively, while $v_{j}$ is the volume fraction of the component $j$ in the mixture.

The EMA works fairly well for low-energy region $(\sim 0$ $5 \mathrm{eV}$ ). It fails at high energy levels because the polarizabilities are not simply added as before, and hence, the charge screening at the interface separating phases should be accounted for. According to this approximation, the components of these films are assumed to be reasonably independent polarizable subunits. Third, the films are presumed to have porous fabric, which suggests that gases can diffuse in or diffuse out.

Applying the EMA on the same suggested mixtures gives

$$
\begin{gathered}
v_{1} \frac{n^{2}\left(\mathrm{a}-\mathrm{Si}_{0.25} \mathrm{C}_{0.75}: \mathrm{H}\right)-n_{\text {eff }}^{2}}{n^{2}\left(\mathrm{a}-\mathrm{Si}_{0.25} \mathrm{C}_{0.75}: \mathrm{H}\right)+2 n_{\text {eff }}^{2}}+v_{2} \frac{n^{2}\left(\mathrm{a}-\mathrm{SiO}_{2}\right)-n_{\text {eff }}^{2}}{n^{2}\left(\mathrm{a}-\mathrm{SiO}_{2}\right)+2 n_{\text {eff }}^{2}}=0, \\
v_{1}+v_{2}=1,
\end{gathered}
$$

where $v_{1}$ and $v_{2}$ are the volume fractions of a- $\mathrm{Si}_{0.25} \mathrm{C}_{0.75}: \mathrm{H}$ and $\mathrm{a}-\mathrm{SiO}_{2}$, respectively. Tables 2 and 3 are a list of the results obtained when applying the EMA on the aged films after 1 and 50 days, respectively. The atomic ratios predicted by the EMA are in agreement with those measured by XPS.

In Figure 4, the refractive index of the underlying TMS layer taken from Table 3 is compared to two untreated plain TMS samples. The effective index of the oxygen-treated TMS film is also shown. It is clear that there is an agreement between the aging of one of the plain TMS samples (221) and the aging of the underlying TMS layer, while the second sample (882) showed a significant difference. This indicates that the plain TMS films may have experienced deposition variations. Furthermore, spectroscopic ellipsometry measurements were done in the spectral range $420 \mathrm{~nm}$ to $700 \mathrm{~nm}$ at an angle of incidence $70^{\circ}$. The effective index of both the top and the TMS layer showed a gradual decrease in time as shown in Figure 5.

\section{Conclusion}

Coordinated XPS, single wavelength ellipsometry, and spectroscopic ellipsometry have been used to study oxygentreated TMS plasma-polymerized films. Ellipsometry measurements show that the refractive index of the samples undergoes a substantial change with time especially in fresh films. Moreover, XPS depth profiling measurements done on these films reveal that the chemical atomic ratios of the constituents change with time. In particular, the strength of the total oxygen signal shows an increase, while the strength of the total carbon signal shows a decrease with time in all samples. The exact reason for this change is not completely understood yet. Similar films are known to have unsaturated free radicals within their fabric. The penetration of oxygen from all atmospheric sources and hence the oxidation of these free radicals is believed to be the main reason for this change.

\section{References}

[1] H. Yasuda, Plasma Polymerization, Academic Press, Orlando, Fla, USA, 1985.

[2] H. Fujiwara, Spectroscopic Ellipsometry Principles and Applications, John Wiley \& Sons, West Sussex, UK, 2007.

[3] D. E. Aspnes, "Expanding horizons: new developments in ellipsometry and polarimetry," Thin Solid Films, vol. 455-456, pp. 3-13, 2004.

[4] T. M. El-Agez, A. A. El Tayyan, and S. A. Taya, "Rotating polarizer-analyzer scanning ellipsometer," Thin Solid Films, vol. 518, no. 19, pp. 5610-5614, 2010.

[5] T. M. El-Agez and S. A. Taya, "An extensive theoretical analysis of the $1: 2$ ratio rotating polarizer-analyzer Fourier ellipsometer," Physica Scripta, vol. 83, Article ID 025701, 2011.

[6] R. M. Azzam and N. M. Bashara, Ellipsometry and Polarized Light, North-Holland, Amsterdam, The Netherlands, 1977.

[7] T. M. El-Agez and S. A. Taya, "A Fourier ellipsometer using rotating polarizer and analyzer at a speed ratio $1: 1$," Journal of Sensors, vol. 2010, Article ID 706829, 7 pages, 2010.

[8] T. M. El-Agez, S. A. Taya, and A. A. El Tayayn, "An improvement of scanning ellipsometer by rotating a polarizer and an analyzer at a speed ratio of 1:3," International Journal of Optomechatronics, vol. 5, no. 1, pp. 51-67, 2011.

[9] P. J. Ozawa, "Organic thin-film capacitor," IEEE Trans. Parts, Mater. Packag., vol. 5, pp. 112-116, 1969.

[10] D. Ristow, "Time-dependent properties of organic thin films deposited by glow discharge," Journal of Materials Science, vol. 12, no. 7, pp. 1411-1415, 1977.

[11] L. S. Tuzov, V. M. Kolotyrkin, and N. N. Tunitskii, "Stability of the dielectric properties of polymer films formed in a glow discharge," International Chemical Engineering, vol. 11, no. 1, pp. 60-64, 1971.

[12] X. Redondas, P. González, B. León, and M. Pérez-Amor, "Dependence on the $\mathrm{C}_{2} \mathrm{H}_{4}$ and $\mathrm{SiH}_{4}$ gas mixture of the Si$\mathrm{C}$ film properties obtained by excimer lamp chemical vapour deposition," Surface and Coatings Technology, vol. 100-101, no. 1-3, pp. 160-163, 1998.

[13] T. M. El-Agez and S. A. Taya, "Development and construction of rotating polarizer analyzer ellipsometer," Optics and Lasers in Engineering, vol. 49, no. 4, pp. 507-513, 2011.

[14] K. Mui, D. K. Basa, F. W. Smith, and R. Corderman, "Optical constants of a series of amorphous hydrogenated 
silicon-carbon alloy films: dependence of optical response on film microstructure and evidence for homogeneous chemical ordering," Physical Review B, vol. 35, no. 15, pp. 8089-8102, 1987.

[15] D. Bruggemann, "Dielectric constant and conductivity of mixtures of isotropic materials," Annals of Physics, vol. 24, no. 7, pp. 636-664, 1935. 

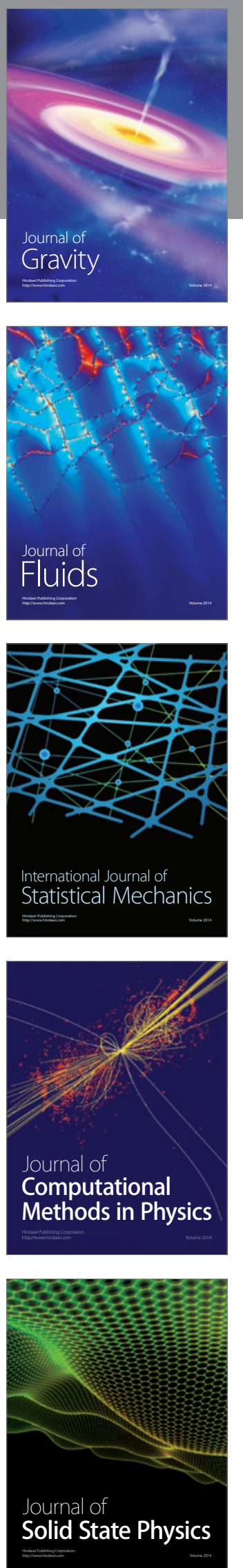

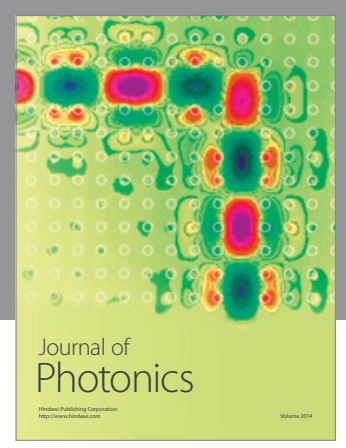

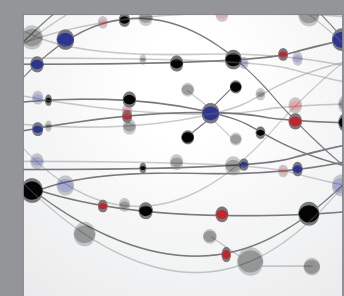

The Scientific World Journal
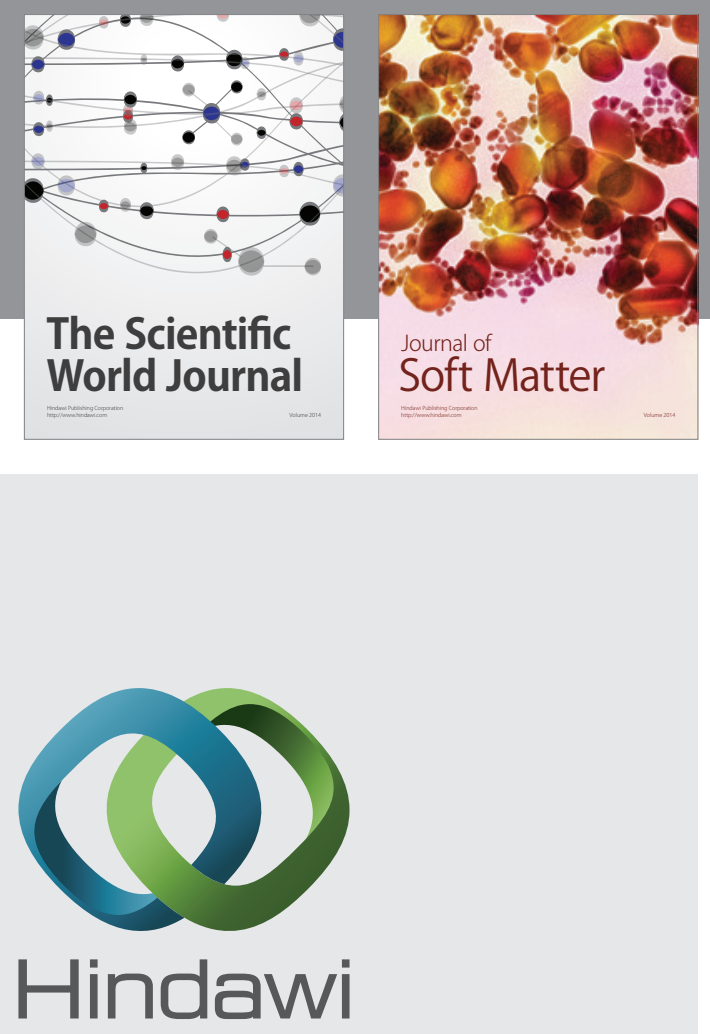

Submit your manuscripts at

http://www.hindawi.com
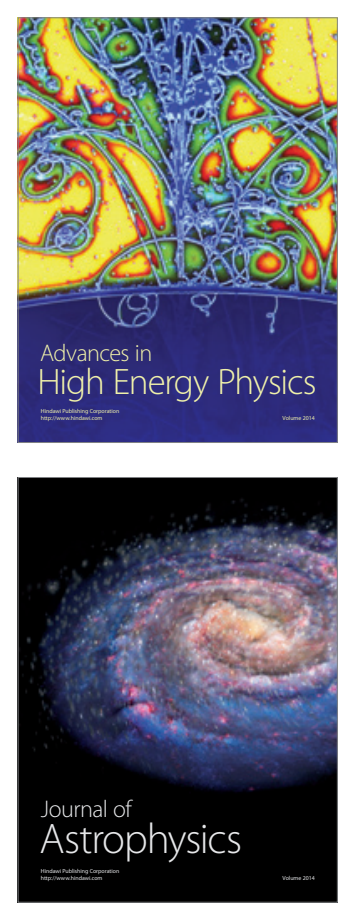
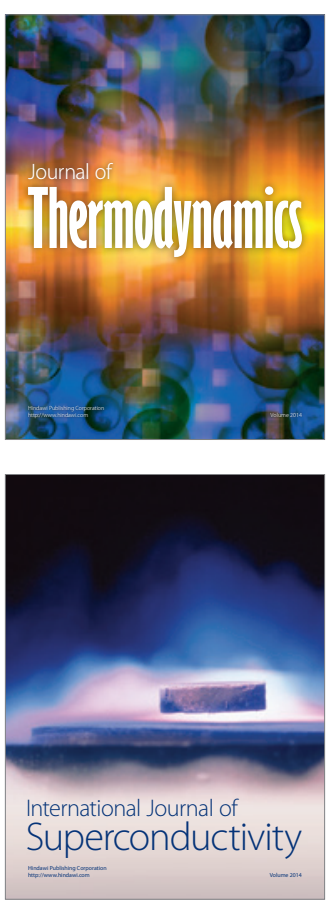
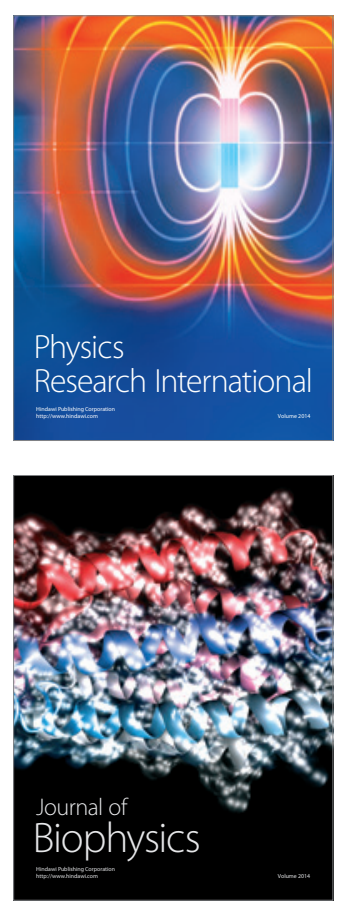
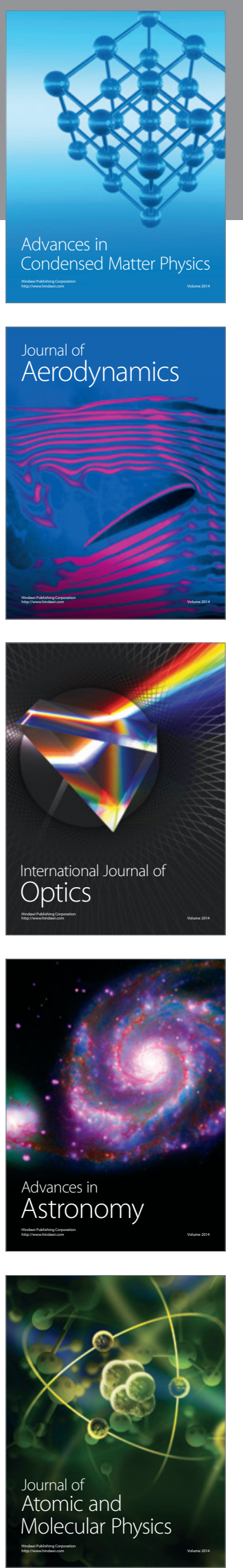